\title{
Detection of vortex sign for scalar speckle fields
}

\author{
Mokhun I. and Galushko Yu.
}

Chernivtsi University, 2 Kotsyubinsky St., Chernivtsi 12, 58012, Ukraine

e-mail: mokhun@itf.cv.net

Received: 05.09.2008

\begin{abstract}
A technique is described for determining vortex sign in the scalar optical fields (including statistical ones) under conditions when it is impossible to use a regular reference beam. The elaborated approach is based on the shift-interferometry technique. The optimal conditions for identifying the vortices are formulated. The results of computer simulations and the corresponding experimental confirmation of our theoretical findings are presented.
\end{abstract}

Keywords: vortex, shift-interferometry, interference forklets, topological charge

PACS: $42.50 . \mathrm{Ct}$

UDC: 535.44

\section{Introduction}

Restoration of phase distribution of optical fields from the analysis of their intensity distribution represents a long-standing optical problem called sometimes as Inverse Source Problem in Optics. Different theoretical and practical aspects of this problem have been considered rather comprehensively (see, e.g., [1]). However, new approaches for solving this problem have been suggested recently on the basis of understanding of special role of amplitude zeroes in the scalar fields [2,3]. As follows from the results [2, 4], the amplitude zeroes are wavefront dislocations (optical vortices), which are combined into some systems, vortex networks. Elements of these networks are connected to each other. Their characteristics define behaviour of the field phase at each its point [3]. As a result, the information about characteristics of the networks and their vortices provides feasibility for restoring the field parameters with high reliability. At the same time, the latter approaches for solving the Inverse Source Problem are still not widely applied, being at the very beginning of development.

One of the aspects of the problem is impossibility of "identification" of the field zeroes. The main characteristic of the optical vortex is its topological charge (sign), defining the direction (clockwise or counter-clockwise) of the phase increasing under bypass of the vortex centre (see, e.g., $[4,5])$. As it is known, complete information on the vortex characteristics may be obtained only from the analysis of data of interferometric experiments [6-9]. At the same time, as a rule, formation of regular reference beams is impossible due to lack of knowledge of pre-history of the field under analysis. 
Thus, elaboration of techniques allowing to obtain information on the vortices' signs for the fields with certain intensity distributions seems to be urgent. Note that in the most general case the fields are to be considered as statistical ones or, in other words, as speckle-fields. On the other hand, we should remind a number of facts important in what follows:

i. Any field after dividing its amplitude enables forming a coherent superposition (see, e.g., [16]). The corresponding components may be easily shifted with respect to each other in the transverse direction.

ii. The phase is practically constant in the speckle centre due to the fact that the phase saddle is positioned in this region [13].

iii. The mean speckle dimension is comparable to the field intensity correlation length (see $[2,14])$.

iv. The field intensity correlation length and the phase one coincide if the field is analyzed in its far-field zone. Moreover, their magnitude coincides with the mean spacing between the absolute intensity minima (or the phase vortices) [2, 14].

v. The intensity networks and the phase ones are linked, at least in the statistical sense. First of all, positions of the absolute intensity minima coincide with the localizations of the centres of phase vortices. The intensity saddle points are preferentially positioned in the areas with relatively large phase gradient [2,10-12]. This allows us to define the field correlation length as a value inversely proportional to the spatial density of the field intensity minima (i.e., their mean spacing) and to indicate the areas the where phase saddles are positioned.

In accordance with the said above, if the field is divided by amplitude on transversely shifted components it can be stated that the areas, where vortices of one of the component are located, coincide with the regions, where the other component has its phase saddle points (i.e., regions where the phase is practically constant). In this case the process of interference of the vortices related to the first component with the other ("shifted") component may be interpreted as a "classical" interference of vortex field structure with a reference plane wave. As a result of this superposition, the corresponding interference forklets appear in the positions of vortices localized in the both interfering components. Naturally, the shift must be less than the mean speckle dimension.

It follows from these facts that one of the solutions of the problem under interest may be application of shift-interferometry techniques (see, e.g., [15]). This paper is thus devoted to application of the shift-interferometry technique for identifying the signs of the vortices.

\section{Method of the vortices signs identification}

The basis of the method elaborated by us is as follows. Obviously, each field may be divided into identical components and one can obtain (see, e.g., [15]) interference of the field with itself. The angle between the components, the shift between them and the ratio of their intensities can be easily controlled. 
Let the field $U(\omega, v)$ be divided into the components $\tilde{U}_{1}(\omega, v)$ and $\tilde{U}_{2}(\omega, v)$. Assume that the component $\tilde{U}_{2}(\omega, v)$ is shifted with respect to the field $\tilde{U}_{1}(\omega, v)$ by the space $\Delta$ in the plane $\omega, v$ in the arbitrary direction. It is natural that the choice of the component $\tilde{U}_{2}(\omega, v)$ to be shifted is quite arbitrary, so that the both components are equivalent in this sense. Moreover, it follows from our further consideration that only the relative component shift is of importance.

Let us assume (without any loss of generality) that the directions of predominant propagation of these fields are different and they are described by the following equations:

$$
\begin{aligned}
& \tilde{U}_{1}(\omega, v)=U_{1}(\omega, v) \exp [j(\alpha \omega+\beta v)] \\
& \tilde{U}_{2}(\omega, v)=U_{2}\left(\omega-\Delta_{\omega}, v-\Delta_{v}\right) \exp [-j(\alpha \omega+\beta v)],
\end{aligned}
$$

where $U_{1}$ и $U_{2}$ are the "exact replicas" of the field $U$ with the corresponding amplitude coefficients determining the mean intensity ratio of the components, the parameters $\alpha=k \sin \alpha^{\prime}$ and $\beta=k \sin \beta^{\prime}$ determine the "incline" of the fields $U_{1}$ and $U_{2}$ with respect to $z$ axis that determines the predominant propagation of the resulting field and $\Delta^{2}=\sqrt{\Delta_{\omega}^{2}+\Delta_{v}^{2}}$ implies the shift of the field $U_{2}$ with respect to the field $U_{1}$.

The intensity of this superposition is described by the following relations:

$$
\begin{aligned}
& J(\omega, v)=J_{1}+J_{2}+ \\
& 2\left\{\left[R_{1} R_{2}+I_{1} I_{2}\right] \cos [2(\alpha \omega+\beta v)]+\left[R_{1} I_{2}-I_{1} R_{2}\right] \sin [2(\alpha \omega+\beta v)]\right\},
\end{aligned}
$$

where $R_{i}, I_{i}(i=1,2)$ denote respectively the real and imaginary parts of the fields $U_{1}$ and $U_{2}$. It is seen from Eq. (2) that the third term in the r. h. s. is zero, whenever $\Delta=0$. As a consequence, the intensity of the resulting field forms an interference pattern containing a set of straight fringes without any discontinuities. Such the pattern is modulated by the intensity of the field $U(\omega, v)$.

The shift $\Delta$ (less than $l_{\text {cor }} / 2$ and more than $l_{c o r} / 10 \div l_{\text {cor }} / 5$ ) between the fields $\tilde{U}_{1}(\omega, v)$ and $\tilde{U}_{2}(\omega, v)$ leads to the situation when the areas of fields containing the vortices superpose with practically plane waves, corresponding to the areas with the phase saddle points. Thus, "canonical" interference forklets appear in the positions of vortices of the fields $U_{1}$ and $U_{2}$ when $\Delta$ is nonzero.

It is worth noticing the following facts:

i. The vortices of opposite signs associated with one of the fields $\left(U_{1}\right.$ or $\left.U_{2}\right)$ form the forklets with different directions.

ii. The directions of the forklets are also different, when the vortices of the same signs are associated with different components. 
iii. The direction of the forklet obtained as a result of interference of the vortex field with a plane reference wave is determined by two factors [9]: the topological charge of the vortex and the mutual orientation of the vortex and the reference waves with respect to the plane of observation.

iv. The normal to the wavefront in the phase saddle points coincides with the predominant direction of wave propagation. Thus, one can state that the information on the predominant directions of propagation of the waves $U_{1}$ and $U_{2}$ unambiguously determines the mutual orientation of the "vortex" and "reference" waves (the field part with a saddle point) with respect to the plane of observation.

It follows from these facts that a control of parameters of the interfering components $U_{1}$ and $U_{2}$ (their propagation directions, relative shift, etc.) would allow determining unambiguously the true value of the topological charge of any field vortex.

It is known that maximal visibility of the interference pattern is achieved when the intensities of the superposing fields are equal. The uniform visibility is impossible when the superposing fields are statistical and the shift $\Delta$ is nonzero. The visibility in the areas with vortices (for example, those associated with the field $U_{1}$ ) tends to the maximal one under the conditions when the intensity $J_{1}$ of one of the fields (the field $U_{1}$ in our case) is significantly larger than the intensity $J_{2}$ of the other component (the field $U_{2}$ ). In other words, favourable conditions for visualising the vortices associated with the field $U_{1}$ would appear due to the fact that the low-intensity parts of the field $U_{1}$ superpose with the parts of the field $U_{2}$ having a similar intensity. At the same time, the interference conditions for the areas with vortices associated with the component $U_{2}$ are extremely unfavourable, even if compare with the other parts of the field $U_{2}$. Only interference forklets corresponding to the non-shifted component must be well observed, when the intensity $J_{1}$ is significantly larger than $J_{2}$. On the contrary, when the intensity $J_{2}$ greatly exceeds $J_{1}$, the vortices of the field $U_{2}$ are visualized.

In such a manner one can determine the positions and signs of the vortices of practically arbitrary fields, provided that the shift $\Delta$ between the components and their intensities $J_{1}$ and $J_{2}$ are controlled.

\section{Computer simulations of speckle-field shift-interferometry}

In this section we present the results of our computer simulations of visualization of the vortices with the aid of speckle field shift-interferometry. The main results are illustrated in Fig. 1 to Fig. 3.

The intensity and the "phase map" of some field area are presented in Fig. 1. It is seen that two adjacent vortices with the opposite topological charges are present within the region under analysis. 


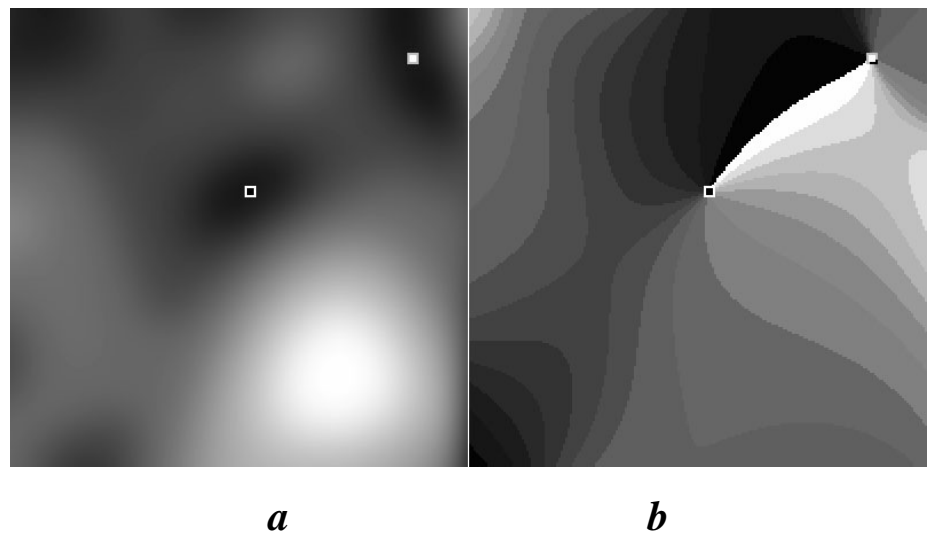

Fig. 1. Spatial distributions of intensity (a) and phase (b) for a fragment of the speckle field. The phase changes from the 0 (white) to $2 \pi$ (black). Gray $x$-like areas in figure (b) are those where the phase saddles are positioned. The two adjacent vortices are depicted by squares. A dark square with a white fringe effect represents a negative vortex and a light square with a black fringe effect - a positive one.

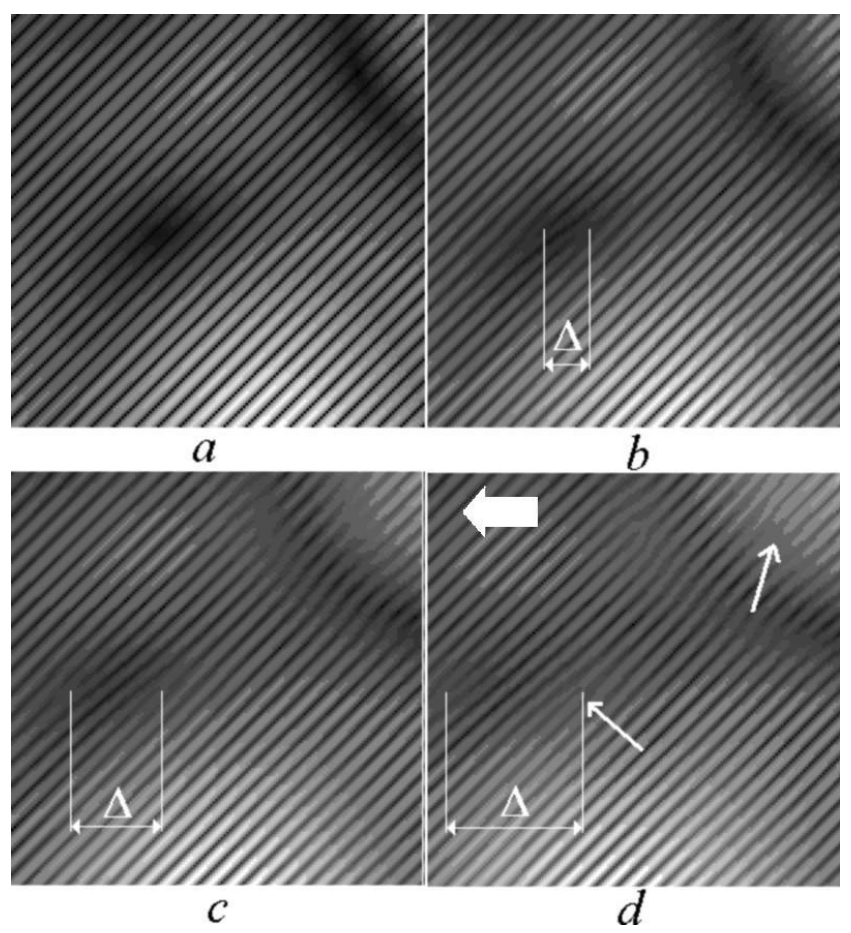

Fig. 2. Shift-interferograms of the speckle field for different magnitudes of the shift $\Delta$ for the field $U_{2}: \mathrm{a}-\Delta=0, \mathrm{~b}-\Delta=0.1 l_{c o r}, \mathrm{c}-\Delta=0.2 l_{c o r}$ and $\mathrm{d}-\Delta=0.3 l_{\text {cor }}$ (see also text). The interference forklets corresponding to the steady field (the field $U_{1}$ ) are indicated by thin white arrows and the direction of the shift of the field $U_{2}$ is shown by thick white arrow. 
The shift-interfrograms for this field area and the shifts $\Delta$ of different magnitudes are shown in Fig. 2. The shift magnitudes for the field $U_{2}$ are denoted in Fig. $2 \mathrm{~b}$ to $2 \mathrm{~d}$. As follows from Fig. 2a, the interference fringes are straight and continuous when $\Delta=0$. As a result, the interference forklets corresponding to the vortices are absent. The pairs of the oppositely directed interference forklets corresponding to the fields $U_{1}$ and $U_{2}$ appear for nonzero shift (see Fig. $2 b$ to $2 d$ ) due to interference of the vortices with smooth parts of these fields. Interference forklets associated with the shift of the component $U_{2}$ relatively to those corresponding to the field $U_{1}$ are in proportion to the increase in the shift $\Delta$.

The results of the shift-interference for different intensity ratios of the fields $U_{1}$ and $U_{2}$ are illustrated in Fig. 3. As follows from Fig. 3c and 3d, only interference forklets corresponding to the field $U_{1}$ are reliably identified in the case when the intensity of the component $U_{1}$ exceeds 20 to 100 times that of the field $U_{2}$.
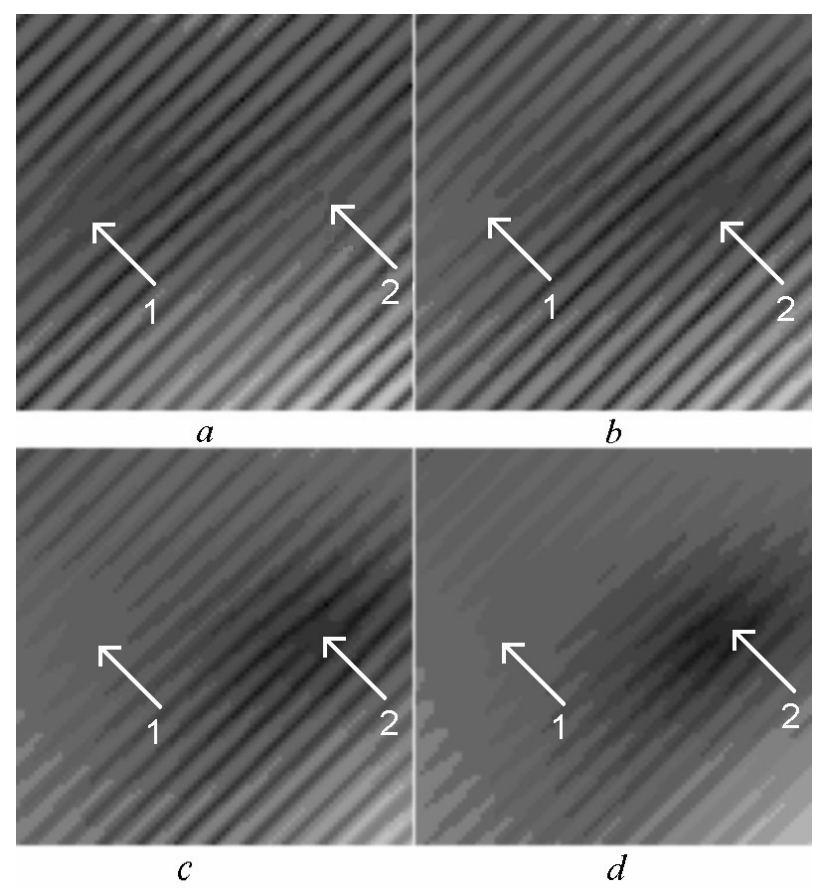

Fig. 3. Shift-interferograms of the speckle field for the shift magnitude $\Delta=0.3 l_{\text {cor }}$ and different ratios of intensities corresponding to the components $U_{1}$ and $U_{2}$ : (a) the mean intensity of the field $U_{1}$ is equal to that of the component $U_{2}$ and it exceeds the intensity of the field $U_{2}$ by 4 (b), (c) 16 and (d) 100 times. Positions of interference forklets formed by the shifted component (the field $U_{2}$ ) are indicated by white arrows 1 and interference forklets corresponding to the steady component (the field $U_{1}$ ) are indicated by white arrows 2 . The shift direction for the field $U_{2}$ is the same as in Fig. 2. 


\section{Experimental disclosure of the vortices' signs using the shift-interferometry}

Experimental apparatus for detection of signs of the vortices in the statistical scalar fields is presented in Fig. 4.

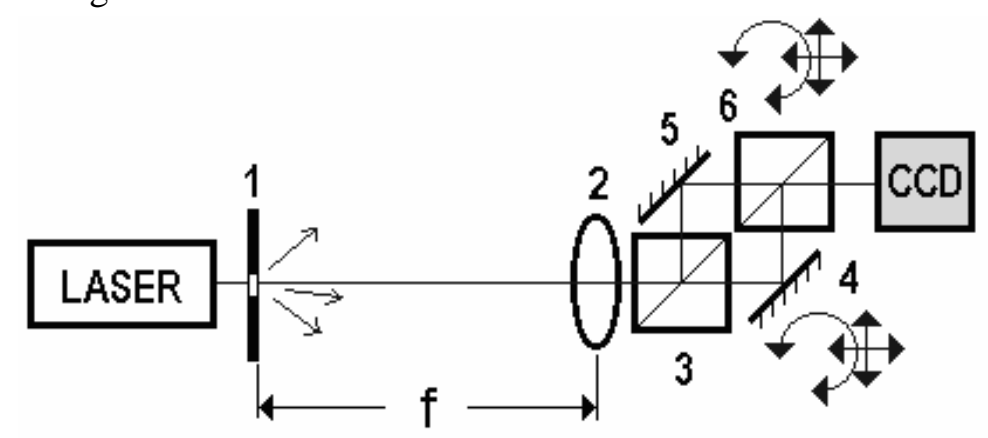

Fig. 4. Experimental setup: 1 - object; 2 - Fourier-transform objective; 3,6 - beam-splitters; 4,5 - mirrors

Non-collimated beam from He-Ne laser illuminates a scattering object 1 (a ground glass screen limited by square hole). The magnitude of the phase variance of the boundary field is chosen in such a manner that the regular component of the scattered field behind the object is absent. The Fourier-transform objective 2 is placed at the focal distance from the object. Due to the latter, the Fourier-transform of the boundary (far) field is observed at the back focal plane of this objective. Mach-Zehnder interferometer 3-6 is placed just behind the objective 2. One of its mirrors, 4 , and an output beam-splitter 6 are mounted on the precision mechanical devices providing fine control of the shift and convergence angle of the field components. Resulting interference patterns and component intensities are fixed by a CCD camera.

The results of the shift-interference are displayed in Fig. 5. Here Fig. 5a corresponds to the zero shift between the field components, while Fig. $5 \mathrm{~b}$ illustrates the interference for the case when the shift between the fields $U_{1}$ and $U_{2}$ amounts approximately half the correlation length (i.e., half the mean dimension of the speckle). The intensity of the steady component exceeds $\sim 10$ times the intensity of the shifted one. As follows from Fig. $5 b$, the vortices of the steady component are easily identified, when compare with those of the shifted one.

It is evident that determination of the vortex characteristics from interference patterns is not so simple problem and, moreover, it always has somewhat subjective character. Nonetheless, we would argue that the signs of practically all, without any exception, vortices of the fields under analysis may be identified with high reliability owing to proper modifications of the ratio of the components' intensities and the shift magnitude between them, even if it is impossible to use a regular reference beam.

Finally, it has to be noted that the technique proposed by us may be also used for some polychromatic fields with "white" vortices. A detailed consideration of the relevant case represents a subject of independent study.

Ukr. J. Phys. Opt. 2008, V9, №4 


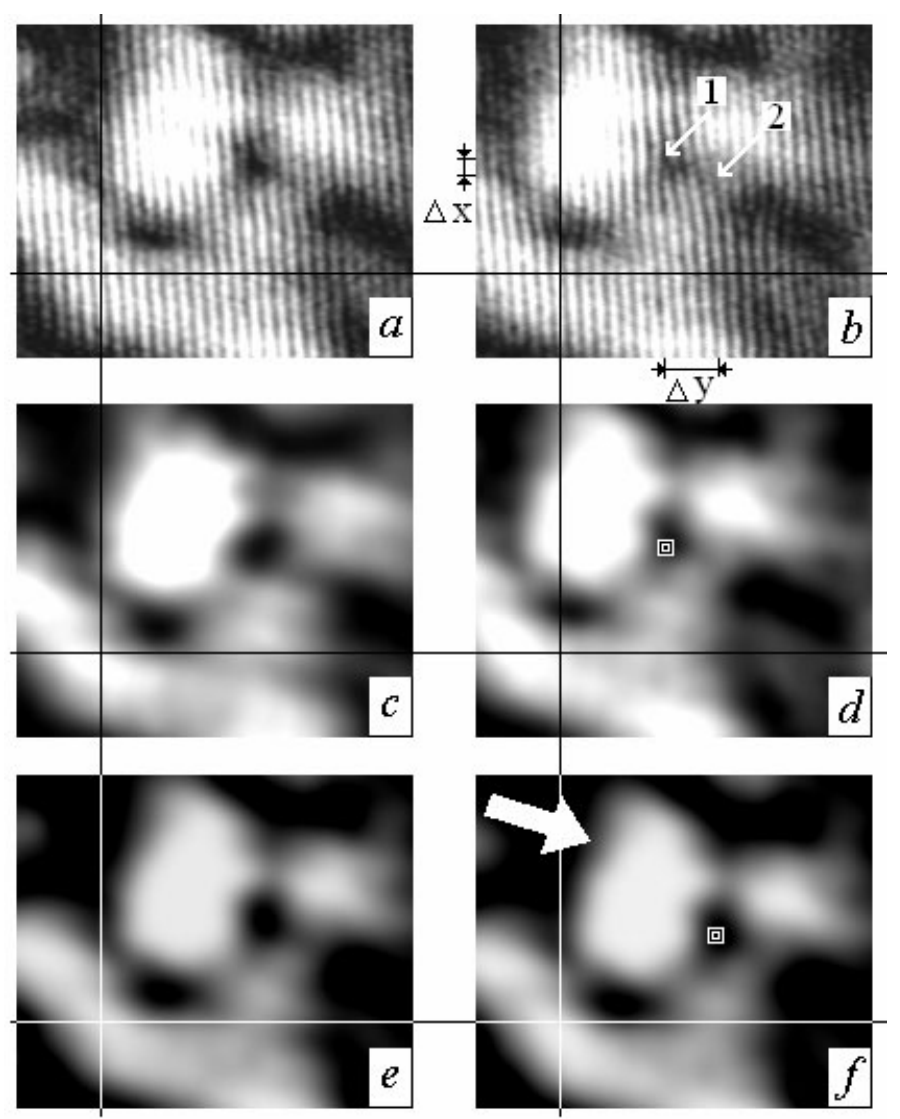

Fig. 5. Results of experimental detection of the vortices' signs using the shift-interferometry: (a) interference pattern of the resulting field for the zero shift between the components and intensity distributions of the steady (c, d) and shifted (e, f) field components, respectively; (b) interference pattern of the resulting field for the shift equal to about half the correlation length. Distances $\Delta x, \Delta y$ illustrate magnitudes of the shifts in the corresponding directions of the coordinate plane. Thin white arrows and numbers in figure (b) indicate the positions of the vortices (interference forklets) corresponding to different field components (1 - the vortex of the steady component, 2 - that of the shifted one). Squares in figures (d), (f) give the positions of the vortices: (d) the vortex of the steady component, ( $f$ ) that of the shifted one. The shift direction for the field $U_{2}$ is denoted in figure (f) by thick white arrow. Straight black and white lines are used as a "coordinate map" for convenient visual perception of the field shifts.

\section{Conclusions}

Summarising the results obtained in the present study, one can draw the following conclusions:

1. The shift-interferometric technique can be used for determining the vortex characteristics of arbitrary fields, including statistical ones.

2. The optimal conditions for the corresponding interferograms are as follows:

(i) the shift between the field components is about $0.3-0.5$ of the field correlation length; 
(ii) the intensity ratio of the components must be chosen so that the intensity of the steady component exceeds 20-50 times that of the shifted component.

\section{References}

1. Baltes H.P. Inverse Source Problems in Optics. Berlin: Springer - Verlag, (1978).

2. Freund I, Shvartsman N, Freilikher V, 1993. Optical dislocation networks in highly random media, Opt. Comm. 101: 247-264.

3. Angelsky O, Brandel R, Mokhun I, 2002. Characteristics of scalar random field and its vortex networks. Recovery of the optical phase. SPIE Proc. 4607: 25-29.

4. Freund I, Shvartsman N, 1994. Wave-field singularities: The sign principle, Phys. Rev. A 50: 5164-5172.

5. Nye J F, F R S, Hajnal J V, Hannay J H, 1988. Phase saddles and dislocations in twodimensional waves such as the tides. Proc. R. Soc. Lond. A 417: 7-20.

6. Baranova N B, Zeldovich B Ya, 1981. Wave front dislocations and amplitude zeroes. JETP 80: 1789-1797.

7. Heckenberg N R, McDuff R, Smith C P, Rubinsztein-Dunlop H, Wegener M J, 1992. Laser beams with phase singularities. Opt. \& Quant. Electr. 24: S951-S962.

8. White A G, Smith C P, Heckenberg N R, Rubinsztein-Dunlop H, McDuff R, Weiss $\mathrm{C} \mathrm{O}, 1991$. Interferometric measurements of phase singularities in the output of a visible laser. J. Mod. Opt. 38: 2531-2541.

9. Basisty I V, Soskin M S, Vasnetsov M V, 1995. Optical wavefront dislocations and their properties. Opt. Comm. 119: 604-612.

10. Freund I, 1998. '1001' correlations in random wave fields. Waves Random Media. 8: 119-158.

11. Freund I, Shvartsman N, 1994. Vortices in Random Wave Fields: Nearest Neibor Anticorrelations. Phys. Rev. Lett. 72: 1008-1011.

12. Mokhun I, 1998. Amplitude zeroes and structure of statistical optical fields. Correlation between the field's intensity and phase. Proc. SPIE 3573: 567-571.

13. Shvartsman N, Freund I, 1995. Speckle spots ride phase saddles sidesaddle. Opt. Comm. 117: 228-234.

14. Baranova N B, Zeldovich B Ya, Mamayev A V, Pilipetsky N F, Shkunov V V, 1982. Investigation of wavefront dislocations density in the light field. JETP 83: 1702-1710.

15. Greivenkamp J.E. and Bruning J.H. Phase Shifting Interferometers. in Optical Shop Testing, D. Malacara, ed., 2nd ed., New York: Wiley (1992).

16. Born M. and Wolf E. Principles of Optics, 6th (corrected) ed. Oxford: Pergamon Press (1987). 